\title{
Long-Term Effects of Intensive Low-Salt Diet Education on Deterioration of Glomerular Filtration Rate among Non-Diabetic Hypertensive Patients with Chronic Kidney Disease
}

\author{
Shin Young Ahn ${ }^{a, b}$ Dong Ki Kim ${ }^{c, d}$ Jung Hwan Park ${ }^{e}$ Sung Joon Shin ${ }^{f}$ \\ Sang Ho Lee ${ }^{g}$ Bum Soon Choi ${ }^{\text {h }}$ Chun Soo Lim ${ }^{i}$ Anna Leej \\ Hyeyoung Jung ${ }^{j}$ Ho Jun Chin ${ }^{d, j, k}$ \\ ${ }^{a}$ Department of Internal Medicine, Korea University Guro Hospital, Seoul, South Korea; \\ ${ }^{b}$ Department of Internal Medicine, Korea University College of Medicine, Seoul, South Korea; \\ 'Department of Internal Medicine, Seoul National University Hospital, Seoul, South Korea; \\ dDepartment of Internal Medicine, Seoul National University College of Medicine, Seoul, South \\ Korea; ${ }^{e}$ Department of Internal Medicine, Konkuk University School of Medicine, Seoul, South

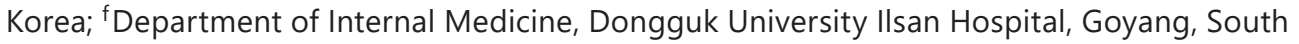 \\ Korea; ${ }^{9}$ Department of Internal Medicine, Kyung Hee University Medical Center, Seoul, South \\ Korea; ${ }^{h}$ Department of Internal Medicine, Eunpyeong St. Mary's Hospital, Seoul, South Korea; \\ 'Department of Internal Medicine, Seoul National University Boramae Medical Center, Seoul, \\ South Korea; jDepartment of Internal Medicine, Seoul National University Bundang Hospital, \\ Seong-Nam, South Korea; ${ }^{k}$ Research Institute of Salt and Health, Seoul, South Korea
}

\section{Keywords}

Education · Low-salt diet · Renal function decline $\cdot$ Chronic kidney disease $\cdot$ Hypertension

\begin{abstract}
Background: Diet modification, especially a decrease in salt intake, might be an important non-pharmacological strategy to improve chronic kidney disease (CKD) prognosis. Objectives: We conducted a prospective cohort study to investigate whether an intensive low-salt diet education program effectively attenuated the rate of renal function decline in hypertensive patients with CKD. Methods: This cohort study recruited 171 participants from a previous open-labelled, case-controlled, randomized clinical trial that originally consisted of 245 hypertensive CKD patients who were assigned to two groups, intensive low-salt diet or conventional education. We evaluated the renal outcomes, which included the rate of change in estimated glomerular filtration rate (eGFR) per year, the increase in serum creatinine $\geq 50 \%$, the decrease in eGFR $\geq 30 \%$, and the percent change in albuminuria throughout the entire study period. Results: The baseline characteristics of the cohort participants between the two groups were similar at the time of trial phase randomization. During the whole study period,
\end{abstract}




\section{Kidney \\ Blood Pressure \\ Research}

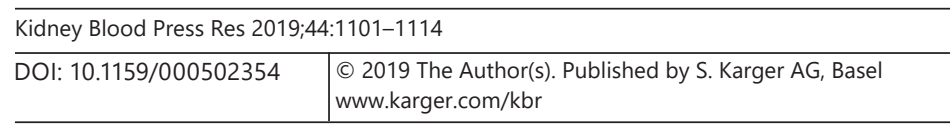

Ahn et al.: Low-Salt Diet Education and Renal Outcome

the rate of renal function decline was significantly faster in the conventional group $(0.11 \pm 4.63$ vs. $-1.53 \pm 3.04 \mathrm{~mL} / \mathrm{min} / 1.73 \mathrm{~m}^{2} /$ year, $\left.p=0.01\right)$. The percent of incremental change in serum creatinine $\geq 50 \%$ was $1.1 \%$ in the intensive group and $8.2 \%$ in the conventional group ( $p=$ 0.025 ), and the percent of decremental change in eGFR $\geq 30 \%$ was $3.3 \%$ in the intensive group and $11.1 \%$ in the conventional group $(p=0.048)$. With logistic regression analysis adjusted for related factors, we found that the conventional group showed a higher risk for deterioration in serum creatinine and eGFR during the entire study period. Especially, we found that the intensive education program preserved eGFR in participants with one, several, or all of the following characteristics at the time of randomization: older age, female, obese, had higher protein intake, higher amounts of albuminuria, higher salt intake. Conclusion: This cohort study demonstrated that an intensive low-salt diet education program attenuated the rate of renal function decline in hypertensive CKD patients independent of its effect on lowering salt intake or albuminuria during the 36 months of follow-up.

(C) 2019 The Author(s)

Published by S. Karger AG, Basel

\section{Introduction}

Chronic kidney disease (CKD) is a risk factor that contributes to cardiovascular morbidity and mortality, hospitalization, and all-cause mortality $[1,2]$. The prevalence of CKD in the United States and Korea has increased during past few decades [3, 4]. The Kidney Disease Improving Global Outcome (KDIGO) guideline recommends pharmacological and non-pharmacological strategies to prevent the progression and complications of CKD. The use of reninangiotensin-aldosterone system inhibitors (RASIs) to reduce albuminuria and control blood pressure is a mainstay pharmacological treatment for the prevention of CKD progression. Diet modification, especially modification of salt intake, might be another way to improve CKD prognosis [5]. In a previous nationwide analysis of epidemiological data of Koreans, we suggested that the prevalence of CKD could be decreased by improved glucose control and blood pressure control, and changing various health-related behaviors, such as the cessation of smoking and avoiding excess intake of salt and protein [6].

There are several randomized controlled trials (RCTs) involving low salt intake in individuals with CKD reduced blood pressure and proteinuria; however, the duration of the studies was too short to test the effect of salt restriction on end points such as CKD progression [7]. Unfortunately, there is a paucity of high-quality prospective RCTs investigating whether low salt intake improves long-term renal outcomes [8]. Post hoc analysis of the RENAAL and IDNT trials and the REIN and REIN-2 trials showed that higher salt intake increased the risk of end-stage renal disease (ESRD) in non-diabetic CKD patients and a composite outcome of doubling serum creatinine and ESRD in diabetic CKD patients using a RASI $[9,10]$. A prospective cohort study showed that lower dietary sodium intake may reduce the rate of decline in estimated glomerular filtration rate (eGFR) in women, although the dietary intake was assessed by food frequency questionnaires [11]. The Trial of Hypertension Prevention (TOHP) I and TOHP II trials were conducted to investigate the long-term effects of low-salt diet education and showed that the risks for mortality, cardiovascular events, and incident hypertension were decreased in the intervention group [12-14]. However, the trials did not measure urinary sodium excretion after 18 months or 36-48 months of the trial phase and did not observe the effect of intervention on renal outcome. Therefore, we conducted a prospective cohort study to investigate whether an intensive low-salt diet education program is effective in reducing the rate of GFR decline among hypertensive CKD patients participating in an open-labelled RCT, which had aimed to determine the effect of short-term intensive low-salt diet education program on albuminuria [15]. 


\section{Kidney \\ Blood Pressure \\ Research}

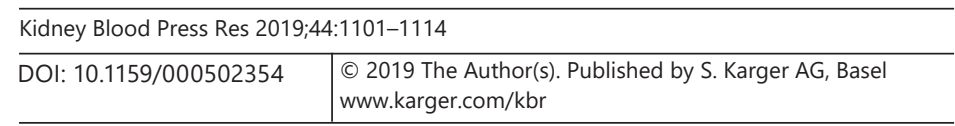

Ahn et al.: Low-Salt Diet Education and Renal Outcome

\section{Materials and Methods}

\section{Study Population}

This study recruited participants from a previous open-labelled, case-controlled, randomized clinical trial (NCT01552954). The inclusion criteria have been described in the previous report [15]. In the trial, all participants were receiving an angiotensin type II receptor blocker (ARB) at random assignment to either intensive low-salt diet education program designed for this study or conventional education. After the trial, almost all of the participants had regular follow-up at outpatient clinics by the same researcher. A few months later, the trial researchers conducted a prospective cohort study with a 1-year duration. So, all subjects enrolled in the trial were asked to participate in the prospective cohort. Among 245 participants who finished the trial, 171 agreed to participate in the prospective cohort. During the trial, all participants were randomly assigned to an intensive or conventional educational group, and they received the low-salt diet education according to the program. During the cohort study, we advised all participants using the same protocol and just observed the laboratory findings related to renal functions or diet intake. We defined the period of the trial as "trial phase, duration of 16 weeks" and the period of the cohort study as "cohort phase, duration of 12 months." Because there was a time interval between the start of the trial and the start of the cohort, the cohort phase was started at $25.1 \pm 4.0$ months later from randomization at the trial phase (Fig. 1). All clinical investigations were conducted in accordance with the 2008 Declaration of Helsinki and the guidelines of good clinical practice. Informed written consent was obtained from each patient before inclusion.

\section{Study Protocol}

The protocol of the trial phase was described in the previous report [15]. We calculated the estimated daily creatinine excretion using Tanaka's equation [16], which confirmed its accuracy [17]. In order to eliminate errors from the incompleteness of 24-h urine collection and difference of muscle mass, we multiplied the 24-h urine variables-to-creatinine ratio (unit/g creatinine) by estimated daily creatinine excretion $(\mathrm{g} / 24 \mathrm{~h}$ ) and obtained the estimated urine excretion of a 24-h urine variable (in unit/24 h) [17]. All participants of the cohort study underwent the first cohort examination and had dietary support by the same clinical dietician with the same protocol; telephone call for 30 min every 4 months during the cohort phase. After the 12-month cohort phase, participants visited the clinic for their second cohort examination (Fig. 1).

\section{Outcome Measures}

The renal outcomes were rate of change in eGFR per year, increase in serum creatinine $\geq 50 \%$, decrease in eGFR $\geq 30 \%$, and percent change in albuminuria during the entire study period. The eGFR was estimated by the Modification of Diet for Renal Disease equation using isotope dilution mass spectrometry-traceable creatinine, which has been previously validated in Koreans $[18,19]$.

\section{Statistics}

All of the analyses were performed using SPSS statistics version 22.0 (IBM corporation, Armonk, NY, USA). Continuous variables were expressed as mean \pm SD, and categorical variables were expressed as frequency (percentage). The Shapiro-Wilk test was used to test for normality of distribution of continuous variables. The independent Student $t$ test or one-way analysis of variance (ANOVA) test was used to compare continuous variables according to the number of subgroups. Pearson's $\chi^{2}$ test or Fisher's exact test was used to analyze the categorical variables according to the number of probability. To assess the effect of low-salt diet 
A.

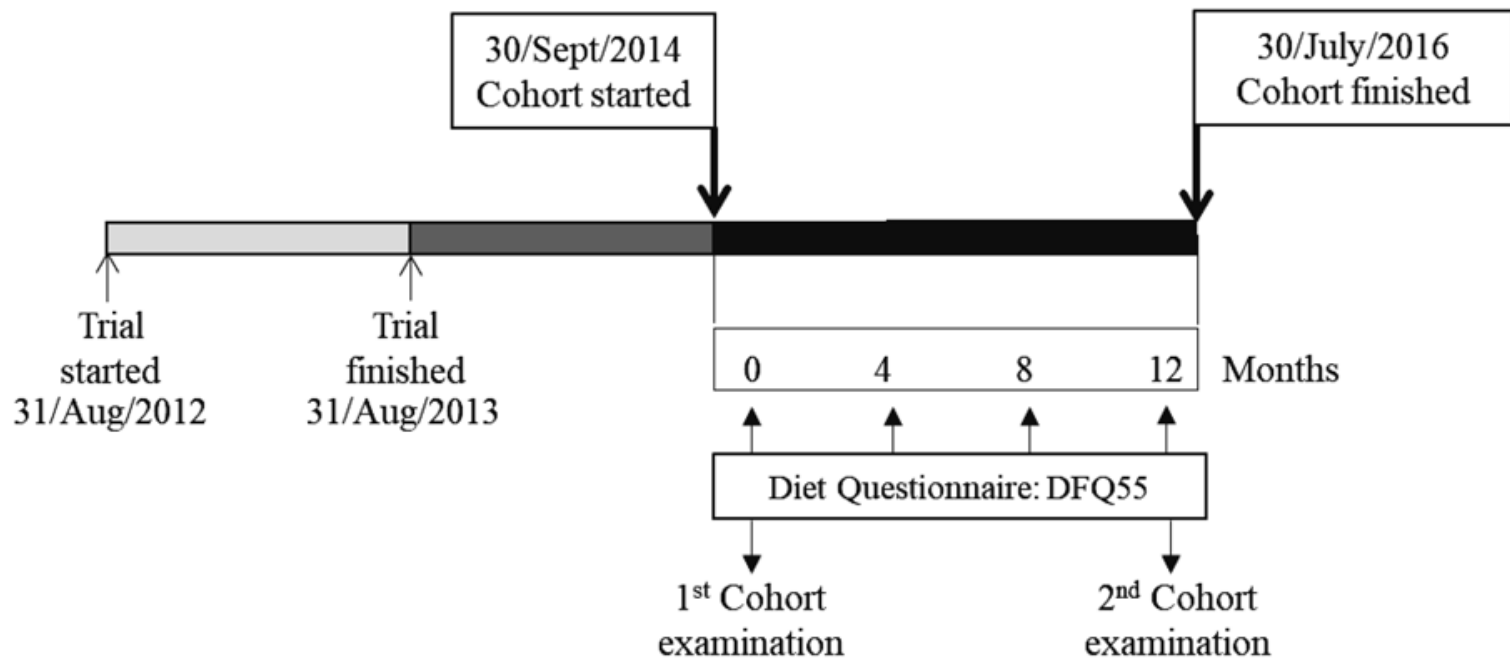

B.

Whole study period

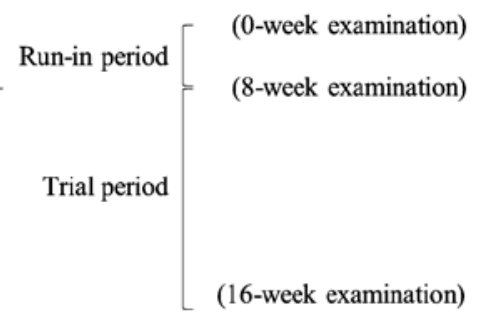

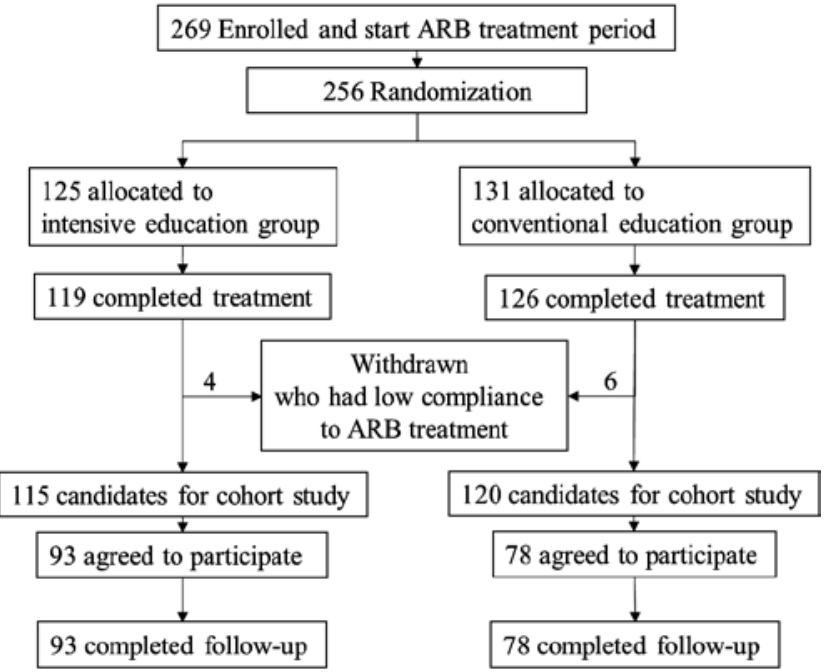

Fig. 1. Schema of this study. A Timeline for the trial phase and cohort phase. The trial phase started on August 31, 2012, and ended on August 31, 2013. The cohort study was initiated on September 30, 2014, and ended on July 30, 2016. Examinations for demographic findings and laboratory tests were conducted at the first and last visits during the cohort phase. During the cohort phase, diet education was given to all participants for 20 min per session through a telephone call with a same clinical dietitian every 4 months. B Study flowchart. Among participants in the trial phase, we excluded 10 patients whose compliance to ARB medication was less than $60 \%$. We enrolled 171 patients out of 235 candidates, all of whom completed the cohort phase. 


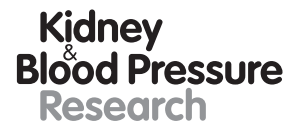

Kidney
Blood Pressure
Research \begin{tabular}{l|l}
\hline Kidney Blood Press Res 2019:44:1101-1114 \\
\hline DOI: 10.1159/000502354 & $\begin{array}{l}\text { @ 2019 The Author(s). Published by S. Karger AG, Basel } \\
\text { www.karger.com/kbr }\end{array}$ \\
\hline
\end{tabular}

Ahn et al.: Low-Salt Diet Education and Renal Outcome

education on blood pressure, albuminuria, urinary sodium excretion, estimated protein intake, and eGFR over time, we used the repeated measures ANOVA test. The relation between variables was estimated with Pearson's correlation coefficient and tested with linear regression analysis for continuous variables and logistic regression analysis for dichotomized variables by adjusting for age, gender, and related factors. Differences with two-tailed $p$ value $<0.05$ were considered statistically significant.

\section{Results}

\section{Patient Characteristics}

Among the 245 patients, 171 (72.8\%) participated in the cohort study. The characteristics of the enrolled patients and those who did not participate in the cohort were not different at the end of trial phase except with regard to compliance with ARB medication and proportion of allocation to intensive education groups (online suppl. Table 1 ; for all online suppl. material, see www.karger.com/doi/10.1159/000502354).

The baseline characteristics of the cohort participants were compared according to the intensive and conventional education group at the time of trial phase randomization. There was no difference in 24-h urine sodium, albumin, urea nitrogen, and creatinine clearance, whether the data were the original data or adjusted by estimated daily urinary creatinine excretion (Table 1). The percentages of compliance for 24-h urine were 98.8 and $93.0 \%$ in the cohort phase, and these values did not differ between the two groups.

This cohort study began enrolling participants at $25.1 \pm 4.0$ months after trial phase randomization. At the time of the cohort enrollment, there were no differences in medication profiles, body weight, blood pressures, eGFR, estimated 24-h urine sodium and albumin excretion rate, and estimated protein intake between groups in the first cohort examination. The second cohort examination was conducted 1 year later. At that time, there were also no differences in parameters between groups; however, eGFR was lower in the conventional group (Table 2). During the 12-month cohort period, participants received $3.9 \pm 0.4$ diet consultations lasting $20.0 \pm 4.0 \mathrm{~min} /$ contact via telephone. The number and duration of consultations were the same in the groups (Table 2).

\section{Renal Outcomes}

At the second cohort examination, the renal function of the intensive group was definitely higher than that of the conventional group $\left(67.0 \pm 29.2\right.$ vs. $57.8 \pm 26.5 \mathrm{~mL} / \mathrm{min} / 1.73 \mathrm{~m}^{2}, p=$ 0.04). During the period from trial phase randomization to the end of the cohort phase, the rate of renal function decline was significantly faster in the conventional group $0.11 \pm 4.63$ vs. $-1.53 \pm 3.04 \mathrm{~mL} / \mathrm{min} / 1.73 \mathrm{~m}^{2} /$ year, $p=0.01$ ) (Table 2 ).

Table 3 showed the changes in clinical parameters at each time interval (end of trial phase, start and end of cohort phase) compared to the time of randomization. As shown by the result of a previous study, the amount of estimated salt intake and albuminuria was more reduced in the intensive group during the trial phase, but the change in eGFR and the slope of eGFR did not differ between the two groups. However, when observed over a longer period of time, the conventional group showed more rapidly reduced renal function (Table 3 ). There was a significant trend in the percent change of eGFR according to each observational period between the two groups ( $p$ for trend $=0.013$ ). The percent changes of eGFR in the two groups were considerably different between the end of the trial phase and the start of the cohort phase (online suppl. Table 2).

Linear regression analysis showed that the rate of eGFR decline during the entire study period from trial randomization to the end of cohort phase was faster in the conventional 
Table 1. Participant characteristics at the time of randomization stratified according to education groups

\begin{tabular}{|c|c|c|c|}
\hline Characteristics & $\begin{array}{l}\text { Intensive group } \\
(n=93)\end{array}$ & $\begin{array}{l}\text { Conventional } \\
\text { group }(n=78)\end{array}$ & $p$ value \\
\hline Age, years & $48.9 \pm 11.4$ & $51.2 \pm 14.2$ & 0.243 \\
\hline Male gender & $44(47.3 \%)$ & $40(51.3 \%)$ & 0.605 \\
\hline Weight, kg & $69.2 \pm 12.1$ & $67.6 \pm 13.5$ & 0.444 \\
\hline Height, $\mathrm{cm}$ & $163.8 \pm 8.8$ & $163.2 \pm 8.7$ & 0.639 \\
\hline Systolic blood pressure, $\mathrm{mm} \mathrm{Hg}$ & $123.8 \pm 13.7$ & $124.4 \pm 14.7$ & 0.766 \\
\hline Diastolic blood pressure, $\mathrm{mm} \mathrm{Hg}$ & $74.9 \pm 11.5$ & $74.5 \pm 9.8$ & 0.823 \\
\hline \multicolumn{4}{|l|}{ Lifestyle } \\
\hline Smoker never/ex/current & $68 / 17 / 8$ & $53 / 16 / 9$ & 0.727 \\
\hline Drinker never/ex/current & $51 / 6 / 36$ & $39 / 8 / 31$ & 0.622 \\
\hline \multicolumn{4}{|l|}{ Blood measurement } \\
\hline $\mathrm{WBC}, / \mathrm{mm}^{3}$ & $6,578 \pm 1,712$ & $6,336 \pm 1,498$ & 0.331 \\
\hline Hemoglobin, g/dL & $13.6 \pm 1.7$ & $13.3 \pm 1.7$ & 0.264 \\
\hline Hematocrit & $40.3 \pm 4.5$ & $39.6 \pm 4.6$ & 0.348 \\
\hline Platelet, $\mathrm{mm}^{3}$ & $235 \pm 54$ & $229 \pm 50$ & 0.451 \\
\hline Creatinine, mg/dL & $1.16 \pm 0.41$ & $1.24 \pm 0.43$ & 0.205 \\
\hline $\mathrm{eGFR}, \mathrm{mL} / \mathrm{min} / 1.73 \mathrm{~m}^{2}$ & $66.3 \pm 24.0$ & $62.1 \pm 25.0$ & 0.266 \\
\hline \multicolumn{4}{|l|}{ Urine measurement } \\
\hline 24-h urine creatinine, $\mathrm{mg} /$ day & $1,240 \pm 410$ & $1,203 \pm 407$ & 0.559 \\
\hline e24-h urine creatinine, mg/day & $1,330 \pm 300$ & $1,292 \pm 312$ & 0.426 \\
\hline 24-h urine $\mathrm{Na}, \mathrm{mEq} /$ day & $165 \pm 76$ & $150 \pm 65$ & 0.180 \\
\hline e24-h urine $\mathrm{Na}, \mathrm{mEq} /$ day & $183 \pm 91$ & $168 \pm 72$ & 0.246 \\
\hline 24-h urine albumin, mg/day & $578 \pm 717$ & $442 \pm 593$ & 0.186 \\
\hline e24-h urine albumin, mg/day & $658 \pm 818$ & $534 \pm 860$ & 0.338 \\
\hline Creatinine clearance, $\mathrm{mL} / \mathrm{min}$ & $82.1 \pm 34.0$ & $72.6 \pm 33.4$ & 0.072 \\
\hline Protein intake, g/kg/day & $0.85 \pm 0.38$ & $0.91 \pm 0.44$ & 0.395 \\
\hline eProtein intake, $\mathrm{g} / \mathrm{kg} /$ day & $0.93 \pm 0.41$ & $0.98 \pm 0.47$ & 0.428 \\
\hline \multicolumn{4}{|l|}{ Comorbidities } \\
\hline Hypertension & $93(100 \%)$ & $78(100 \%)$ & 1.000 \\
\hline Dyslipidemia & $50(54.9 \%)$ & $46(63.9 \%)$ & 0.249 \\
\hline Coronary artery disease & $1(1.1 \%)$ & $0(0.0 \%)$ & 0.356 \\
\hline Stroke & $2(2.2 \%)$ & $2(2.6 \%)$ & 0.895 \\
\hline \multicolumn{4}{|l|}{ Medications at baseline examination } \\
\hline Angiotensin converting enzyme blocker & $0(0 \%)$ & $0(0 \%)$ & $\mathrm{UC}$ \\
\hline Angiotensin II receptor blocker & $100.0(100.0 \%)$ & $100.0(100.0 \%)$ & UC \\
\hline Beta-blocker & $26(28.0 \%)$ & $19(24.4 \%)$ & 0.595 \\
\hline Calcium channel blocker & $37(39.8 \%)$ & $27(34.6 \%)$ & 0.487 \\
\hline Diuretics & $0(0.0 \%)$ & $1(1.3 \%)$ & 0.273 \\
\hline Other hypertension medications & $13(14.0 \%)$ & $7(9.0 \%)$ & 0.310 \\
\hline Anti-lipid medications & $48(51.6 \%)$ & $43(55.1 \%)$ & 0.646 \\
\hline Anti-platelet medications & $40(43.0 \%)$ & $40(51.3 \%)$ & 0.280 \\
\hline Steroids & $2(2.2 \%)$ & $1(1.3 \%)$ & 0.667 \\
\hline Immunosuppressive agents & $0(0.0 \%)$ & $1(1.3 \%)$ & 0.273 \\
\hline Nonsteroidal anti-inflammatory drugs & $4(4.3 \%)$ & $4(5.1 \%)$ & 0.799 \\
\hline \multicolumn{4}{|l|}{ Compliance to ARB medication at trial } \\
\hline phase, $\%$ & $95.2 \pm 8.0$ & $96.1 \pm 6.9$ & 0.408 \\
\hline
\end{tabular}

WBC, white blood cell; eGFR, estimated glomerular filtration rate calculated by MDRD equation using IDMS-traceable serum creatinine; e24-h urine creatinine, $-2.04 \times$ age $+14.89 \times$ weight $(\mathrm{kg})+16.14 \times$ height (cm) - 2,244.45; e24-h urine $\mathrm{Na}$, estimated 24-h urine sodium excretion by adjustment of measured 24-h urine sodium based on the estimated daily creatinine amount by Tanaka's equation; e24-h urine albumin, estimated 24-h urine albumin excretion by adjustment of measured 24-h urine albumin based on the estimated daily creatinine amount by Tanaka's equation; eProtein intake, estimated daily protein intake calculated from adjusted 24-h urine urea nitrogen based on the estimated daily creatinine amount by Tanaka's equation; ARB, angiotensin II receptor blocker; UC, unable to calculate. 
Table 2. Characteristics of participants at the cohort examinations

\begin{tabular}{|c|c|c|c|}
\hline & $\begin{array}{l}\text { Intensive group } \\
(n=93)\end{array}$ & $\begin{array}{l}\text { Conventional } \\
\text { group }(n=78)\end{array}$ & $p$ value \\
\hline \multicolumn{4}{|l|}{ At the first cohort examination } \\
\hline Follow-up after randomization, months & $25.2 \pm 3.8$ & $25.0 \pm 4.2$ & 0.841 \\
\hline Age, years & $50.7 \pm 11.6$ & $52.8 \pm 14.2$ & 0.289 \\
\hline Male gender & $44(47.3 \%)$ & $40(51.3 \%)$ & 0.605 \\
\hline Weight, kg & $69.3 \pm 12.7$ & $66.9 \pm 11.7$ & 0.193 \\
\hline Height, $\mathrm{cm}$ & $164.0 \pm 8.6$ & $163.3 \pm 8.8$ & 0.569 \\
\hline Systolic blood pressure, $\mathrm{mm} \mathrm{Hg}$ & $127.6 \pm 14.4$ & $126.2 \pm 12.9$ & 0.521 \\
\hline Diastolic blood pressure, $\mathrm{mm} \mathrm{Hg}$ & $77.1 \pm 11.4$ & $76.2 \pm 8.8$ & 0.546 \\
\hline Creatinine, $\mathrm{mg} / \mathrm{dL}$ & $1.17 \pm 0.49$ & $1.29 \pm 0.51$ & 0.120 \\
\hline $\mathrm{eGFR}, \mathrm{mL} / \mathrm{min} / 1.73 \mathrm{~m}^{2}$ & $67.2 \pm 25.9$ & $60.2 \pm 24.7$ & 0.075 \\
\hline Slope of eGFR change, $\mathrm{mL} / \mathrm{min} / 1.73 \mathrm{~m}^{2} /$ year & $0.23 \pm 5.05$ & $-1.45 \pm 4.95$ & 0.031 \\
\hline e24-h urine $\mathrm{Na}, \mathrm{mEq} /$ day & $184 \pm 107$ & $174 \pm 77$ & 0.494 \\
\hline e24-h urine albumin, mg/day & $615 \pm 743$ & $612 \pm 1059$ & 0.982 \\
\hline eProtein intake, g/kg/day & $1.24 \pm 0.50$ & $1.31 \pm 0.69$ & 0.421 \\
\hline \multicolumn{4}{|l|}{ Medication } \\
\hline Angiotensin II receptor blocker & $83(89.2 \%)$ & $57(73.1 \%)$ & 0.006 \\
\hline Angiotensin converting enzyme blocker & $3(3.2 \%)$ & $9(11.5 \%)$ & 0.034 \\
\hline Renin-angiotensin-aldosterone blocker & $85(91.4 \%)$ & $66(84.6 \%)$ & 0.169 \\
\hline Beta-blocker & $25(26.9 \%)$ & $14(17.9 \%)$ & 0.166 \\
\hline Calcium channel blocker & $28(30.1 \%)$ & $26(33.3 \%)$ & 0.651 \\
\hline Diuretics & $1(1.1 \%)$ & $3(3.8 \%)$ & 0.232 \\
\hline Other hypertension medications & $0(0.0 \%)$ & $1(1.3 \%)$ & 0.273 \\
\hline Statin & $47(50.5 \%)$ & $42(53.8 \%)$ & 0.666 \\
\hline Steroids & $4(4.3 \%)$ & $1(1.3 \%)$ & 0.377 \\
\hline Immunosuppressive agents & $1(1.1 \%)$ & $1(1.3 \%)$ & 0.900 \\
\hline Nonsteroidal anti-inflammatory drugs & $1(1.1 \%)$ & $0(0.0 \%)$ & 0.358 \\
\hline \multicolumn{4}{|l|}{ At the second cohort examination } \\
\hline Follow-up period after randomization, months & $36.4 \pm 3.9$ & $36.3 \pm 4.4$ & 0.850 \\
\hline Mean education time, min/education & $20.4 \pm 3.9$ & $19.6 \pm 4.1$ & 0.211 \\
\hline Age, years & $51.5 \pm 11.6$ & $54.2 \pm 14.3$ & 0.213 \\
\hline Gender, male & $44(47.3 \%)$ & $40(51.3 \%)$ & 0.605 \\
\hline Weight, kg & $69.4 \pm 12.4$ & $67.8 \pm 12.1$ & 0.403 \\
\hline Height, $\mathrm{cm}$ & $164.0 \pm 8.7$ & $162.8 \pm 9.1$ & 0.373 \\
\hline Systolic blood pressure, $\mathrm{mm} \mathrm{Hg}$ & $126.5 \pm 11.7$ & $127.1 \pm 14.3$ & 0.784 \\
\hline Diastolic blood pressure, $\mathrm{mm} \mathrm{Hg}$ & $76.0 \pm 9.4$ & $75.8 \pm 9.8$ & 0.923 \\
\hline Creatinine, $\mathrm{mg} / \mathrm{dL}$ & $1.22 \pm 0.63$ & $1.41 \pm 0.69$ & 0.064 \\
\hline $\mathrm{eGFR}, \mathrm{mL} / \mathrm{min} / 1.73 \mathrm{~m}^{2}$ & $67.0 \pm 29.2$ & $57.8 \pm 26.5$ & 0.040 \\
\hline Slope of eGFR change, $\mathrm{mL} / \mathrm{min} / 1.73 \mathrm{~m}^{2} /$ year & $0.11 \pm 4.63$ & $-1.53 \pm 3.04$ & 0.010 \\
\hline e24-h urine $\mathrm{Na}, \mathrm{mEq} /$ day & $162 \pm 71$ & $147 \pm 81$ & 0.212 \\
\hline e24-h urine albumin, mg/day & $720 \pm 1061$ & $553 \pm 745$ & 0.251 \\
\hline eProtein intake, g/kg/day & $1.09 \pm 0.43$ & $1.12 \pm 0.54$ & 0.705 \\
\hline \multicolumn{4}{|l|}{ Medication } \\
\hline Angiotensin II receptor blocker & $82(90.1 \%)$ & $55(74.3 \%)$ & 0.007 \\
\hline Angiotensin converting enzyme blocker & $2(2.2 \%)$ & $10(13.5 \%)$ & 0.005 \\
\hline Renin-angiotensin-aldosterone blocker & $84(92.3 \%)$ & $65(87.8 \%)$ & 0.335 \\
\hline Beta-blocker & $23(25.3 \%)$ & $12(16.2 \%)$ & 0.157 \\
\hline Calcium channel blocker & $27(29.7 \%)$ & $23(31.1 \%)$ & 0.845 \\
\hline Diuretics & $2(2.2 \%)$ & $2(2.7 \%)$ & 0.834 \\
\hline Other hypertension medications & $0(0.0 \%)$ & $1(1.4 \%)$ & 0.266 \\
\hline
\end{tabular}

eGFR, estimated glomerular filtration rate calculated by MDRD equation using IDMS-traceable serum creatinine; e24-h urine $\mathrm{Na}$, estimated 24-h urine sodium excretion by adjustment of measured 24-h urine sodium based on the estimated daily creatinine amount by Tanaka's equation; e24-h urine albumin, estimated 24-h urine albumin excretion by adjustment of measured 24-h urine albumin based on the estimated daily creatinine amount by Tanaka's equation; eProtein intake, estimated daily protein intake calculated from adjusted 24-h urine urea nitrogen based on the estimated daily creatinine amount by Tanaka's equation; slope of eGFR change, annual change rate of eGFR during the follow-up period after randomization; ARB, antiotensin II receptor blocker. 


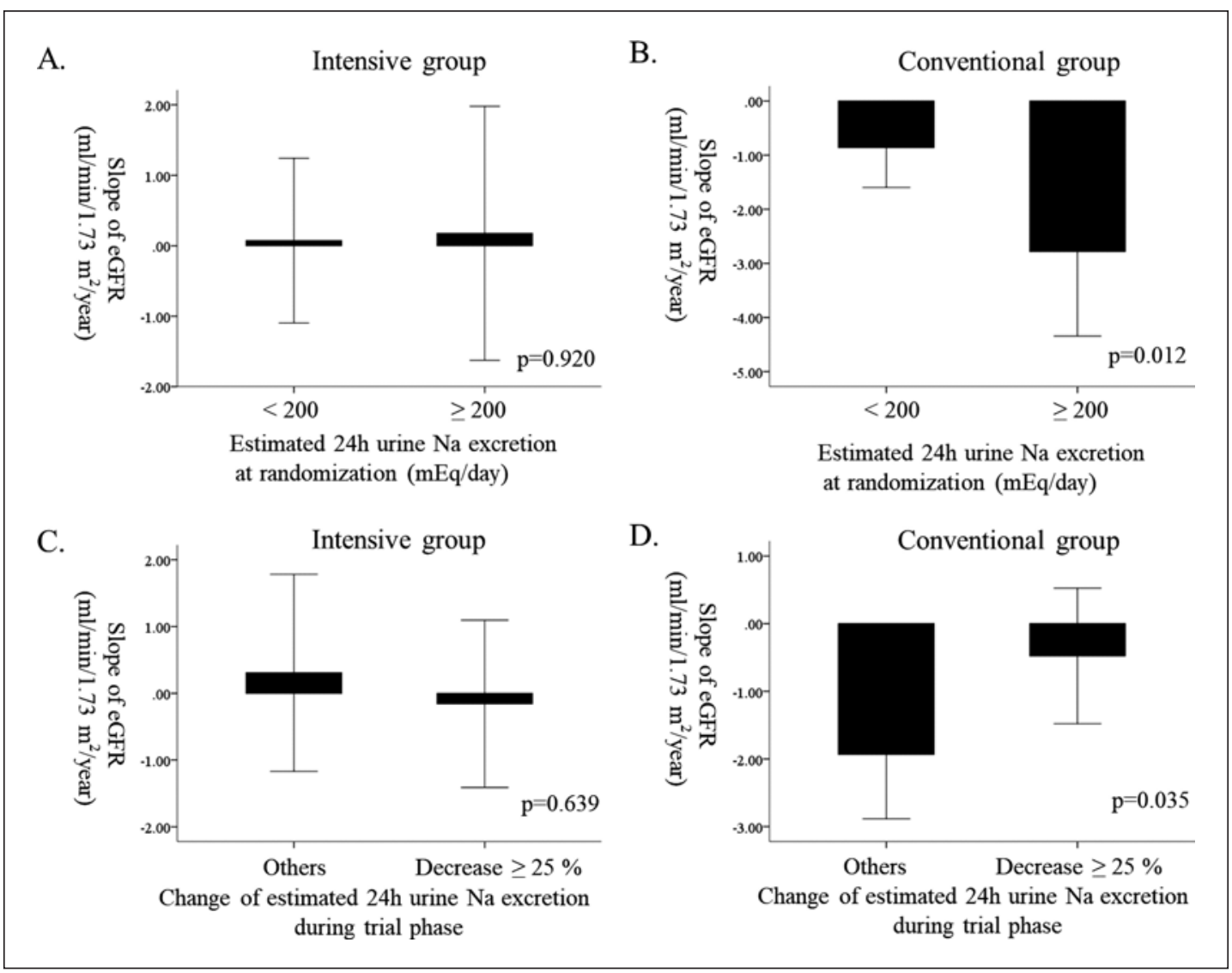

Fig. 2. Slope of eGFR during whole trial and observational period according to randomization groups. A, B Slope of eGFR according to the level of 24-h urine Na excretion at randomization period in each randomization group. C, D Slope of eGFR according to the change in 24-h urine Na excretion during the 16-week trial period in each randomization group.

group than in the intensive group after adjustment for age, gender, and factors known to be related to the change in eGFR rate (Table 4). There was no interaction between education group and the amount of 24-h urinary sodium or albumin excretion.

The percent of incremental change in serum creatinine $\geq 50 \%$ was $1.1 \%$ in the intensive group and $8.2 \%$ in the conventional group (Fisher's exact test, $p=0.025$ ), and the percent of decremental change in eGFR $\geq 30 \%$ was $3.3 \%$ in the intensive group and $11.1 \%$ in the conventional group (Fisher's exact test, $p=0.048$ ). We evaluated whether diet education was an independent risk factor for each dichotomized end point, where logistic regression adjusted for related factors and demonstrated that the conventional group showed higher risk for deterioration in serum creatinine and eGFR during the entire study period (Table 5).

We evaluated the difference in the rate of change of eGFR in various subgroups according to intervention. Intensive education program was related to preserving eGFR in participants who were older, female, obese, had higher protein intake $\geq 0.8 \mathrm{~g} / \mathrm{kg} /$ day, higher amount of albuminuria $\geq 1,000 \mathrm{mg} /$ day, and higher salt intake $\geq 200 \mathrm{mEq} /$ day at the time of randomization. Furthermore, that program was effective for preserving eGFR in participants who did not achieve reduction in salt intake during the trial phase (Table 6).

We analyzed the effect of urinary sodium excretion on the change in eGFR in each education group (Fig. 2). In the intensive group, the amount of urinary sodium excretion 
Table 3. Changes in clinical parameters at each time interval compared to the time of randomization

\begin{tabular}{|c|c|c|c|c|}
\hline & $\begin{array}{l}\text { Intensive } \\
\text { group }\end{array}$ & $\begin{array}{l}\text { Conventional } \\
\text { group }\end{array}$ & $\begin{array}{l}\text { Differ- } \\
\text { ence of } \\
\text { mean }\end{array}$ & $p$ value \\
\hline \multicolumn{5}{|l|}{ Change in e24-h urine $\mathrm{Na}, \mathrm{mEq} /$ day } \\
\hline 16-week examination-randomization & $-40 \pm 78$ & $-4 \pm 63$ & -35 & 0.002 \\
\hline 1st cohort examination-randomization & $1.4 \pm 111$ & $8.2 \pm 82$ & -7 & 0.659 \\
\hline 2nd cohort examination-randomization & $-24 \pm 80$ & $-15 \pm 68$ & -10 & 0.430 \\
\hline \multicolumn{5}{|l|}{ Change in e24-h albumin, mg/day } \\
\hline 16-week examination-randomization & $-208 \pm 410$ & $-36 \pm 425$ & -172 & 0.009 \\
\hline 1st cohort examination-randomization & $-43 \pm 551$ & $88 \pm 615$ & -130 & 0.151 \\
\hline 2nd cohort examination-randomization & $71 \pm 752$ & $90 \pm 515$ & -20 & 0.852 \\
\hline \multicolumn{5}{|l|}{ Change in eProtein intake, $\mathrm{g} / \mathrm{kg} /$ day } \\
\hline 16-week examination-randomization & $0.03 \pm 0.32$ & $-0.02 \pm 0.35$ & 0.05 & 0.382 \\
\hline 1st cohort examination-randomization & $0.31 \pm 0.64$ & $0.34 \pm 0.75$ & -0.03 & 0.791 \\
\hline 2nd cohort examination-randomization & $0.17 \pm 0.59$ & $0.13 \pm 0.61$ & 0.03 & 0.722 \\
\hline \multicolumn{5}{|l|}{ Change in SBP, $\mathrm{mm} \mathrm{Hg}$} \\
\hline 16-week examination-randomization & $-2.1 \pm 13.9$ & $-1.7 \pm 15.5$ & -0.4 & 0.872 \\
\hline 1st cohort examination-randomization & $3.8 \pm 15.8$ & $1.8 \pm 15.9$ & 2.0 & 0.411 \\
\hline 2nd cohort examination-randomization & $2.9 \pm 15.9$ & $3.1 \pm 16.9$ & -0.16 & 0.950 \\
\hline \multicolumn{5}{|l|}{ Change in $\mathrm{DBP}, \mathrm{mm} \mathrm{Hg}$} \\
\hline 16-week examination-randomization & $-0.8 \pm 9.8$ & $0.6 \pm 9.7$ & -1.5 & 0.327 \\
\hline 1st cohort examination-randomization & $2.3 \pm 11.1$ & $1.7 \pm 11.0$ & 0.6 & 0.728 \\
\hline 2nd cohort examination-randomization & $1.7 \pm 11.0$ & $1.5 \pm 12.1$ & -0.4 & 0.839 \\
\hline \multicolumn{5}{|l|}{ Change in eGFR, $\mathrm{mL} / \mathrm{min} / 1.73 \mathrm{~m}^{2}$} \\
\hline 16-week examination-randomization & $-1.1 \pm 8.4$ & $-0.0 \pm 6.9$ & -9.5 & 0.389 \\
\hline 1st cohort examination-randomization & $0.6 \pm 9.9$ & $-2.3 \pm 9.9$ & 3.0 & 0.055 \\
\hline 2nd cohort examination-randomization & $0.1 \pm 12.8$ & $-4.5 \pm 9.2$ & 4.6 & 0.012 \\
\hline \multicolumn{5}{|l|}{ Slope of eGFR, $\mathrm{mL} / \mathrm{min} / 1.73 \mathrm{~m}^{2} /$ year } \\
\hline 16-week examination-randomization & $-12.72 \pm 83.20$ & $0.77 \pm 74.17$ & -0.16 & 0.269 \\
\hline 1st cohort examination-randomization & $0.23 \pm 5.05$ & $-1.45 \pm 4.95$ & 1.68 & 0.031 \\
\hline 2nd cohort examination-randomization & $0.11 \pm 4.63$ & $-1.53 \pm 3.04$ & 1.64 & 0.010 \\
\hline
\end{tabular}

16-week examination-randomization, changes in parameters during 8 weeks between the randomization period and the 16-week follow-up period; 1st cohort examination-randomization, changes in parameters between the randomization period and the first cohort examination period; 2nd cohort examinationrandomization, changes in parameters between the randomization period and the second cohort examination period; e24-h urine Na, estimated 24-h urine sodium excretion by adjustment of measured 24-h urine sodium based on the estimated daily creatinine amount by Tanaka's equation; e24-h urine albumin, estimated 24-h urine albumin excretion by adjustment of measured 24-h urine albumin based on the estimated daily creatinine amount by Tanaka's equation; eProtein intake, estimated daily protein intake calculated from adjusted 24-h urine urea nitrogen based on the estimated daily creatinine amount by Tanaka's equation; eGFR, glomerular filtration rate calculated by the MDRD equation using IDMS-traceable creatinine; slope of eGFR, difference in eGFR between two observational periods divided by follow-up duration as year-unit; SBP, systolic blood pressure; DBP, diastolic blood pressure; $p$ value was estimated by Student $t$ test.

at randomization or change in urinary sodium excretion during the trial phase did not influence the eGFR change rate during the entire study period, whereas the average amount of 24-h urine albumin had a negative relation with the eGFR change rate conline suppl. Table 3). In the conventional group, participants with higher urinary sodium excretion ( $\geq 200 \mathrm{mEq} /$ day) at randomization showed a rapid decline in eGFR during the 
Table 4. Factors related to rate of change in eGFR during the whole study period

\begin{tabular}{lllll}
\hline Parameters & $B$ & $95 \%$ CI of B & VIF $^{\mathrm{a}}$ & $p$ value \\
\hline $\begin{array}{l}\text { Group of randomization (conventional group) } \\
\text { e24-h urine albumin at randomization }\end{array}$ & -1.737 & $-2.985 ;-0.490$ & 1.012 & 0.007 \\
$\quad(\geq 1,000$ mg/day) & -0.878 & $-1.689 ;-0.068$ & 1.012 & 0.034 \\
\hline
\end{tabular}

Linear regression model adjusted by age, gender, and eGFR at randomization, and related factors to the slope of eGFR change, such as, estimated 24-h urine albumin and group of randomization among parameters at the randomization period. The number of participants included in this model was 168 out of 171. VIF, variance inflation factor. ${ }^{a}$ Group of randomization compared to intensive education group.

Table 5. Conventional education as a risk for the increase in serum creatinine $\geq 50 \%$ and decrease in eGFR $\geq 30 \%$ during the whole study period

\begin{tabular}{llrll}
\hline & Wald & RR & $95 \%$ CI for RR & $p$ value \\
\hline For increase in creatinine $\geq 50 \%{ }^{\mathrm{a}}$ & 3.908 & 10.273 & $1.020 ; 103.433$ & 0.048 \\
For decrease in eGFR $\geq 30 \%^{\mathrm{b}}$ & 3.469 & 5.559 & $0.914 ; 33.800$ & 0.063
\end{tabular}

${ }^{a}$ Logistic regression for increase in creatinine $\geq 50 \%$ was adjusted for age, and related factors, such as, gender, 24-h urine albumin excretion and serum creatinine at the randomization period, and group of education.

${ }^{b}$ Logistic regression for decrease in eGFR $\geq 30 \%$ was adjusted for gender, related factors, such as, age, group of 24-h urine albumin excretion, group of estimated protein intake, and eGFR at the randomization period, and group of education. Group of 24-h urine albumin excretion: group 1, 24-h urine albumin $<1,000$ $\mathrm{mg}$ /day; group 2, 24-h urine albumin $\geq 1,000 \mathrm{mg} /$ day. Group of estimated protein intake: group 1, estimated protein intake $<1.2 \mathrm{~g} / \mathrm{kg} /$ day; group 2 , estimated protein intake $\geq 1.2 \mathrm{~g} / \mathrm{kg} /$ day.

study period ( $p=0.012$ ), and participants with effective decrease in urinary sodium excretion (decline $\geq 25 \%$ of sodium excretion) during the trial phase showed a reduced rate of decline in eGFR ( $p=0.035)$ compared to the others. The participants in the conventional education group showed high variability in the slope of eGFR during the entire study period. In the conventional group, 24-h urine sodium and albumin excretion at randomization and change in 24-h urine sodium excretion during the trial phase showed a negative relationship with the change in eGFR during the entire study period (online suppl. Table 3).

\section{Discussion}

Especially, we found that the intensive low-salt diet education program designed for this study independently delayed the rate of eGFR decline over the 36-month period of the study. And the program preserved eGFR in participants with one, several, or all of the following characteristics at the time of randomization: older, female, obese, with higher protein intake, higher amounts of albuminuria, higher salt intake. Our study also showed that higher salt intake induced a more rapid decline in eGFR in participants that received conventional low-salt diet education. 
Table 6. The effect of an intensive diet education on change in GFR during the observational period in each subgroup

\begin{tabular}{|c|c|c|c|}
\hline Parameters & Intensive group & Conventional group & $p$ value \\
\hline \multicolumn{4}{|l|}{ Age at randomization } \\
\hline$<50$ years & $0.28 \pm 5.11$ & $-1.24 \pm 3.33$ & 0.133 \\
\hline$\geq 50$ years & $-0.06 \pm 4.14$ & $-1.08 \pm 2.76$ & 0.031 \\
\hline \multicolumn{4}{|l|}{ Gender } \\
\hline Female & $0.17 \pm 0.64$ & $-2.04 \pm 3.21$ & 0.005 \\
\hline Male & $0.03 \pm 5.56$ & $-1.00 \pm 2.80$ & 0.324 \\
\hline \multicolumn{4}{|c|}{ BMI at randomization } \\
\hline$<25 \mathrm{~kg} / \mathrm{m}^{2}$ & $-0.39 \pm 3.55$ & $-0.92 \pm 2.65$ & 0.467 \\
\hline$\geq 25 \mathrm{~kg} / \mathrm{m}^{2}$ & $0.48 \pm 5.30$ & $-2.14 \pm 3.31$ & 0.010 \\
\hline \multicolumn{4}{|c|}{ eProtein intake at randomization } \\
\hline$<0.8 \mathrm{~g} / \mathrm{kg} /$ day & $0.71 \pm 5.98$ & $-1.17 \pm 3.23$ & 0.223 \\
\hline$\geq 0.8 \mathrm{~g} / \mathrm{kg} /$ day & $-0.11 \pm 4.07$ & $-1.60 \pm 2.99$ & 0.029 \\
\hline \multicolumn{4}{|c|}{ e24-h urine albumin at randomization } \\
\hline$<1,000 \mathrm{mg} /$ day & $0.07 \pm 4.56$ & $-0.86 \pm 2.54$ & 0.206 \\
\hline$\geq 1,000$ mg/day & $0.18 \pm 4.82$ & $-2.78 \pm 3.61$ & 0.017 \\
\hline \multicolumn{4}{|c|}{ e24-h urine Na at randomization } \\
\hline$<200 \mathrm{mEq} /$ day & $0.07 \pm 4.56$ & $-0.86 \pm 2.53$ & 0.206 \\
\hline$\geq 200 \mathrm{mEq} /$ day & $0.17 \pm 4.82$ & $-2.78 \pm 3.61$ & 0.017 \\
\hline \multicolumn{4}{|c|}{ Change in e24-h urine Na during trial phase } \\
\hline Others & $0.31 \pm 5.19$ & $-1.93 \pm 3.31$ & 0.012 \\
\hline Decrease $\geq 25 \%$ & $-0.16 \pm 3.92$ & $-0.48 \pm 2.14$ & 0.736 \\
\hline \multicolumn{4}{|c|}{$\begin{array}{l}\text { Slope of eGFR change (mL/min/ } 1.73 \mathrm{~m}^{2} / \text { year) during the entire observational period was compared } \\
\text { between groups in each subgroup at the randomization period. BMI, body mass index; eProtein intake, } \\
\text { estimated daily protein intake calculated from adjusted } 24 \text {-h urine urea nitrogen based on the estimated } \\
\text { daily creatinine amount by Tanaka's equation; e24-h urine albumin, estimated } 24 \text {-h urine albumin excretion } \\
\text { by adjustment of measured } 24 \text {-h urine albumin based on the estimated daily creatinine amount by Tanaka's } \\
\text { equation; e24-h urine Na, estimated } 24 \text {-h urine sodium excretion by adjustment of measured } 24 \text {-h urine } \\
\text { sodium based on the estimated daily creatinine amount by Tanaka's equation; change in e24-h urine Na } \\
\text { during trial phase, percent change in e24-h urine Na during the } 16 \text {-week trial period. }\end{array}$} \\
\hline
\end{tabular}

Several studies have evaluated the benefits of low-salt diet in CKD patients. One metaanalysis showed that reducing salt intake significantly reduced systolic and diastolic blood pressure, and proteinuria [7]. In the study, there were no changes in body weight, serum creatinine, creatinine clearance, eGFR, and serum total cholesterol. However, the duration of the studies was too short to test the effect of salt restriction on endpoints such as mortality, cardiovascular events, or CKD progression [7]. The clinical end point of CKD is renal failure or ESRD; however, it may take a long time to identify this outcome. The rate of decline in GFR over long intervals is also accepted as a measure of kidney disease progression because the time until kidney failure depends on the current level of GFR and the subsequent rate of GFR decline [20]. The planning committee supported by the National Kidney Foundation of the United States proposed that a decline in GFR of 30\% could be a valid surrogate end point for progression to ESRD in a clinical trial of CKD and recommended a follow-up during the trial of at least 2-3 years to allow a thorough evaluation of interventions [20]. In this study, patients were followed up for a mean duration of 36.3 months, and our outcomes were rate of eGFR 


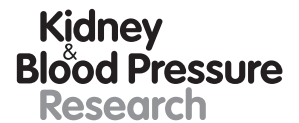

Kidney
Blood Pressure

Research \begin{tabular}{l|l}
\hline Kidney Blood Press Res 2019;44:1101-1114 \\
\hline DOI: 10.1159/000502354 & $\begin{array}{l}\text { @ 2 2019 The Author(s). Published by S. Karger AG, Basel } \\
\text { www.karger.com/kbr }\end{array}$ \\
\hline
\end{tabular}

Ahn et al.: Low-Salt Diet Education and Renal Outcome

decline per year, eGFR decrease $\geq 30 \%$, and increase in serum creatinine $\geq 50 \%$. We found that the intervention was effective in delaying renal function deterioration.

With respect to evaluating the long-term effect of salt intake, there have been two post hoc analyses. One of the post hoc analyses demonstrated that higher salt intake is associated with increased risk of progression to ESRD in patients with non-diabetic CKD who received an angiotensin-converting enzyme inhibitor [10]. In another trials of Hypertension Prevention (TOHP) I and II, 3,126 participants were randomized to intervention or usual care of education and counseling on reducing salt intake for a duration of 18 months (TOHP I) or 36-48 months (TOHP II). The net reduction in 24-h urine sodium excretion was $44 \mathrm{mEq}$ in TOHP I [21] and $33 \mathrm{mEq}$ in TOHP II [22]. After 10-15 years of follow-up, the risk for a cardiovascular event was $25 \%$ lower in the intervention group among participants in the TOHP I and II trials [12].

The observational follow-up included $77.3 \%$ of participants in the original TOHP trials, which is a similar participation rate as in this study. However, the observational follow-up study did not collect 24-h urine samples to evaluate whether the effect of lowering salt intake is persistent and is related to long-term outcomes with a causal relationship. One clinical center participated in TOHP I and followed 181 men and women; this center measured body weight and 24-h urine sodium excretion, and showed that there were no differences in the change of weight and 24-h urine sodium excretion from baseline during the 7-year follow-up period. However, the incidence of hypertension was lower in the weight loss intervention group ( $p=0.02)$ and tended to be lower in the sodium reduction active intervention group. After 7 years, the effects of the intervention on body weight and urinary sodium excretion disappeared, but the beneficial effect on risk of hypertension was evident [14]. We found a similar effect of urinary sodium reduction on renal function. Intensive low-salt diet education for 8 weeks resulted in a decrease in 24-h urine sodium excretion by $35 \mathrm{mEq}$ and a decrease in albuminuria during the trial phase, and this difference vanished at the mean follow-up of 25.1 months after randomization. However, differences in the change in eGFR, slope of eGFR, and proportion of eGFR decrease $\geq 30 \%$ and increase in serum creatinine $\geq 50 \%$ between the two groups were evident at the end of cohort phase-mean follow-up duration of 36.3 months after randomization of trial phase (Table 3). Especially, the slope of eGFR change of the conventional group was similar to that of hypertensive patients, whereas the slope of eGFR change of the intensive group was lower than that of hypertensive patients in another study (Table 1) [23]. The amount of 24-h urine sodium excretion at randomization and change in 24-h urine sodium excretion during the trial phase did not seem to be a risk factor for GFR progression among all participants, but in the conventional low-salt diet education group, these two factors were significantly related to the rate of decline in eGFR. Therefore, reduction of 24-h urine sodium excretion during the trial phase by intensive low-salt diet education is expected to influence the change in eGFR during the follow-up period. It is possible that intensive low-salt diet education had other effects which were not directly measured but affected renal function. For example, because diets include many ingredients other than sodium or protein, education aimed at reducing sodium intake changes the participant's attitude towards dietary lifestyle, although we could not verify these effects in this study. Indeed, we also found that urine potassium excretion was more reduced in the intensive group during the entire study period (data not shown).

This study has some limitations worth noting. First, this study did not intend to prove the effect of low-salt diet education on GFR decline and might not have sufficient statistical power for it. Second, we did not include all participants in the trial phase; nevertheless, the inclusion rate in this cohort study was similar to that in the observational TOHP study. The participants enrolled in the cohort study were more compliant to ARB treatment and were more frequently allocated to the intensive education group in the trial phase, which might be a confounding factor affecting the final results of this cohort study. Third, during the cohort phase, an ARB 
was used more frequently in the intensive education group, while an angiotensin-converting enzyme inhibitor was used more frequently in the conventional education group. These differences may have had a confounding effect on the eGFR change, although the overall rate of RASI usage was not different between groups. Fourth, the study population consisted of non-diabetic hypertensive Korean patients. And we did not include all subjects in the trial phase; therefore, the results of this study could not be generalized to the general hypertensive CKD population and other ethnicities because of race differences. Finally, we could not explain the exact mechanism whereby intensive low-salt diet education slowed the rate of eGFR in this study.

\section{Conclusion}

Short-term intensive low-salt diet education attenuated the rate of renal function decline in hypertensive CKD patients independent of its effects on lowering salt intake or albuminuria. The effect was more prominent in patients with higher risks of CKD progression, such as those who were older, female, obese, had higher protein intake, higher amount of albuminuria, and higher salt intake. Therefore, healthcare providers should be informed of the advantages of intensive low-salt diet education, and these programs should be incorporated into daily practice.

\section{Acknowledgement}

The authors would like to thank Hyunjin Cho for excellent data collection.

\section{Statement of Ethics}

All clinical investigations were conducted in accordance with the 2008 Declaration of Helsinki and the guidelines of good clinical practice. The protocol was approved by the Institutional Review Board (IRB) of Seoul National University Bundang Hospital (IRB No. B-1405/251-007). Informed written consent was obtained from each patient before inclusion.

\section{Disclosure Statement}

The authors declare no conflicts of interest.

\section{Funding Sources}

This study was funded by Daiichi Sankyo Korea Co. Ltd. and Seoul National University Bundang Hospital (grant No.: 02-2015-046).

\section{Author Contributions}

All authors have contributed to the manuscript and approved its submission. 


\section{Kidney \\ Blood Pressure \\ Research}

\begin{tabular}{l|l}
\hline Kidney Blood Press Res 2019;44:1101-1114 \\
\hline DOI: 10.1159/000502354 & $\begin{array}{l}\text { @ 2019 The Author(s). Published by S. Karger AG, Basel } \\
\text { www.karger.com/kbr }\end{array}$ \\
\hline
\end{tabular}

\section{References}

1 Go AS, Chertow GM, Fan D, McCulloch CE, Hsu CY. Chronic kidney disease and the risks of death, cardiovascular events, and hospitalization. N Engl J Med. 2004 Sep;351(13):1296-305.

2 Foley RN, Murray AM, Li S, Herzog CA, McBean AM, Eggers PW, et al. Chronic kidney disease and the risk for cardiovascular disease, renal replacement, and death in the United States Medicare population, 1998 to 1999. J Am Soc Nephrol. 2005 Feb;16(2):489-95.

3 Coresh J, Selvin E, Stevens LA, Manzi J, Kusek JW, Eggers P, et al. Prevalence of chronic kidney disease in the United States. JAMA. 2007 Nov;298(17):2038-47.

4 Kim S, Lim CS, Han DC, Kim GS, Chin HJ, Kim SJ, et al. The prevalence of chronic kidney disease (CKD) and the associated factors to CKD in urban Korea: a population-based cross-sectional epidemiologic study. J Korean Med Sci. 2009 Jan;24 Suppl:S11-21.

5 Stevens PE, Levin A; Kidney Disease: Improving Global Outcomes Chronic Kidney Disease Guideline Development Work Group Members. Evaluation and management of chronic kidney disease: synopsis of the kidney disease: improving global outcomes 2012 clinical practice guideline. Ann Intern Med. 2013 Jun;158(11):82530.

6 Lee SW, Kim YC, Oh SW, Koo HS, Na KY, Chae DW, et al. Trends in the prevalence of chronic kidney disease, other chronic diseases and health-related behaviors in an adult Korean population: data from the Korean National Health and Nutrition Examination Survey (KNHANES). Nephrol Dial Transplant. 2011 Dec;26(12): 3975-80.

7 McMahon EJ, Campbell KL, Bauer JD, Mudge DW. Altered dietary salt intake for people with chronic kidney disease. Cochrane Database Syst Rev. 2015 Feb;(2):CD010070.

8 Lambers Heerspink HJ, Navis G, Ritz E. Salt intake in kidney disease-a missed therapeutic opportunity? Nephrol Dial Transplant. 2012 Sep;27(9):3435-42.

9 Lambers Heerspink HJ, Holtkamp FA, Parving HH, Navis GJ, Lewis JB, Ritz E, et al. Moderation of dietary sodium potentiates the renal and cardiovascular protective effects of angiotensin receptor blockers. Kidney Int. 2012 Aug;82(3):330-7.

10 Vegter S, Perna A, Postma MJ, Navis G, Remuzzi G, Ruggenenti P. Sodium intake, ACE inhibition, and progression to ESRD. J Am Soc Nephrol. 2012 Jan;23(1):165-73.

11 Lin J, Hu FB, Curhan GC. Associations of diet with albuminuria and kidney function decline. Clin J Am Soc Nephrol. 2010 May;5(5):836-43.

12 Cook NR, Cutler JA, Obarzanek E, Buring JE, Rexrode KM, Kumanyika SK, et al. Long term effects of dietary sodium reduction on cardiovascular disease outcomes: observational follow-up of the trials of hypertension prevention (TOHP). BMJ. 2007 Apr;334(7599):885-8.

13 Cook NR, Appel LJ, Whelton PK. Sodium Intake and All-Cause Mortality Over 20 Years in the Trials of Hypertension Prevention. J Am Coll Cardiol. 2016 Oct;68(15):1609-17.

14 He J, Whelton PK, Appel LJ, Charleston J, Klag MJ. Long-term effects of weight loss and dietary sodium reduction on incidence of hypertension. Hypertension. 2000 Feb;35(2):544-9.

15 Hwang JH, Chin HJ, Kim S, Kim DK, Kim S, Park JH, et al. Effects of intensive low-salt diet education on albuminuria among nondiabetic patients with hypertension treated with olmesartan: a single-blinded randomized, controlled trial. Clin J Am Soc Nephrol. 2014 Dec;9(12):2059-69.

16 Tanaka T, Okamura T, Miura K, Kadowaki T, Ueshima H, Nakagawa H, et al. A simple method to estimate populational 24-h urinary sodium and potassium excretion using a casual urine specimen. J Hum Hypertens. 2002 Feb;16(2):97-103.

17 Chin HJ, Kim DK, Park JH, Shin SJ, Lee SH, Choi BS, et al. Effect of urine urea nitrogen and protein intake adjusted by using the estimated urine creatinine excretion rate on the antiproteinuric effect of angiotensin II type I receptor blockers. Nutrition. 2015 Nov-Dec;31(11-12):1333-8.

18 Lee CS, Cha RH, Lim YH, Kim H, Song KH, Gu N, et al. Ethnic coefficients for glomerular filtration rate estimation by the Modification of Diet in Renal Disease study equations in the Korean population. J Korean Med Sci. 2010 Nov; 25(11):1616-25.

19 Oh YJ, Cha RH, Lee SH, Yu KS, Kim SE, Kim H, et al. Validation of the Korean coefficient for the modification of diet in renal disease study equation. Korean J Intern Med (Korean Assoc Intern Med). 2016 Mar;31(2):344-56.

20 Levey AS, Inker LA, Matsushita K, Greene T, Willis K, Lewis E, et al. GFR decline as an end point for clinical trials in CKD: a scientific workshop sponsored by the National Kidney Foundation and the US Food and Drug Administration. Am J Kidney Dis. 2014 Dec;64(6):821-35.

21 Whelton PK. The effects of nonpharmacologic interventions on blood pressure of persons with high normal levels. Results of the Trials of Hypertension Prevention, Phase I. JAMA. 1992 Mar;267(9):1213-20.

22 The Trials of Hypertension Prevention Collaborative Research Group. Effects of weight loss and sodium reduction intervention on blood pressure and hypertension incidence in overweight people with high-normal blood pressure. The Trials of Hypertension Prevention, phase II. Arch Intern Med. 1997 Mar;157(6):657-67.

23 Hanratty R, Chonchol M, Miriam Dickinson L, Beaty BL, Estacio RO, Mackenzie TD, et al. Incident chronic kidney disease and the rate of kidney function decline in individuals with hypertension. Nephrol Dial Transplant. 2010 Mar;25(3):801-7. 